Annales Academiæ Scientiarum Fennicæ

Mathematica

Volumen 37, 2012, 635-648

\title{
HANKEL OPERATORS ON LARGE WEIGHTED BERGMAN SPACES
}

\author{
Petros Galanopoulos and Jordi Pau \\ Aristotle University of Thessaloniki, School of Mathematics \\ 54124 Thessaloniki, Greece; galanopoulos_petros@yahoo.gr \\ Universitat de Barcelona, Departament de Matemàtica Aplicada i Analisi \\ Gran Via de les Corts Catalanes, 585, 08007 Barcelona, Spain; jordi.pau@ub.edu
}

\begin{abstract}
We completely describe the boundedness, compactness and membership in Schatten $p$-classes of the (big) Hankel operator with conjugate analytic symbols on large weighted Bergman spaces.
\end{abstract}

\section{Introduction and main results}

The theory of Hankel operators on the classical Hardy space is an important area of mathematical analysis, and a lot of applications in different domains of mathematics have been found: interpolation problems, rational approximation, stationary processes or perturbation theory. An account of all of that can be found, for example, in [21] and [22]. The development of the theory of Hankel operators led to different generalizations of the original concept. A lot of progress has been made in the study of Hankel operators in other spaces of analytic functions: Bergman spaces on the disc [3], [6], [16], Dirichlet-type spaces [25], [26], Bergman spaces in the unit ball of $\mathbf{C}^{N}$ [4], [28], or even in bounded symmetric domains [27]. In the present paper, we study Hankel operators acting on weighted Bergman spaces on the unit disc with radial rapidly decreasing weights (see below for the definition). More concretely, we describe the boundedness, compactness and membership in Schatten classes of big Hankel operators with conjugate analytic symbols in terms of function theoretical properties of its symbol.

Let $\mathbf{D}$ be the unit disc in the complex plane, and denote by $H(\mathbf{D})$ the space of all analytic functions in $\mathbf{D}$. A positive function $w(r), 0 \leq r<1$, which is integrable in $(0,1)$, will be called a weight function. We extend $w$ to $\mathbf{D}$ setting $w(z)=w(|z|)$, $z \in \mathbf{D}$. The weighted Bergman space $A^{2}(w)$ consists of those analytic functions $f$ on the unit disc $\mathbf{D}$ with

$$
\|f\|_{w}=\left(\int_{\mathbf{D}}|f(z)|^{2} w(z) d m(z)\right)^{1 / 2}<\infty,
$$

where $d m(z)=\frac{1}{\pi} d x d y$ is the normalized area measure on $\mathbf{D}$. The space $A^{2}(w)$ is a reproducing kernel Hilbert space: for each $z \in \mathbf{D}$, there are functions $K_{z} \in A^{2}(w)$ with $f(z)=\left\langle f, K_{z}\right\rangle_{w}$, where $\langle f, g\rangle_{w}=\int_{\mathbf{D}} f(z) \overline{g(z)} w(z) d m(z)$ is the usual inner

doi:10.5186/aasfm.2012.3739

2010 Mathematics Subject Classification: Primary 30H20, 47B10, 47B35.

Key words: Bergman spaces, Hankel operators, Schatten classes.

The second author is partially supported by SGR grant 2009SGR 420 (Generalitat de Catalunya) and DGICYT grant MTM2011-27932-C02-01 (MCyT/MEC). 
product in $L^{2}(w):=L^{2}(\mathbf{D}, w d m)$. The orthogonal projection from $L^{2}(w)$ to $A^{2}(w)$ is given by

$$
\operatorname{Pf}(z)=\int_{\mathbf{D}} f(\zeta) \overline{K_{z}(\zeta)} w(\zeta) d m(\zeta)
$$

Let $g \in A^{2}(w)$. The (big) Hankel operator on $A^{2}(w)$ with conjugate analytic symbol $\bar{g}$ is defined by

$$
H_{\bar{g}} f=\bar{g} f-P(\bar{g} f), \quad f \in A^{2}(w) .
$$

Note that, since we are considering only radial weights, using the same argument as in the unweighted Bergman space, one can show that the polynomials are dense in $A^{2}(w)$ (note that this does not necessarily holds for non radial weights. An example is given in [13, Chapter 9, Exercise 13], and also in [10, p. 138]). Thus, if $H^{\infty}$ denotes the algebra of all bounded analytic functions on the unit disc, the Hankel operator is well defined in the dense subset $H^{\infty}$ of $A^{2}(w)$.

We will restrict our study to a certain class $\mathcal{W}$ of radial rapidly decreasing weights. These weights are going to decrease faster than any standard weight $\left(1-r^{2}\right)^{\alpha}$, and the corresponding weighted Bergman space $A^{2}(w)$ are large spaces in the sense that it contains all the standard Bergman spaces $A_{\alpha}^{2}$, for $\alpha>-1$. Concretely, we consider a decreasing weight of the form $w(z)=e^{-\varphi(z)}$, where $\varphi \in C^{2}(\mathbf{D})$ is a radial function such that $(\Delta \varphi(z))^{-1 / 2} \asymp \tau(z)$, for a radial positive function $\tau(z)$ that decreases to 0 as $|z| \rightarrow 1^{-}$, and $\lim _{r \rightarrow 1^{-}} \tau^{\prime}(r)=0$. Here $\Delta$ denotes the standard Laplace operator. Furthermore, we shall also suppose that either there exist a constant $C>0$ such that $\tau(r)(1-r)^{-C}$ increases for $r$ close to 1 or

$$
\lim _{r \rightarrow 1^{-}} \tau^{\prime}(r) \log \frac{1}{\tau(r)}=0 .
$$

If the weight $w$ satisfies all the previous conditions, we shall say that the weight $w$ belongs to the class $\mathcal{W}$. Typical examples of weights in the class $\mathcal{W}$ are the exponential type weights

$$
w_{\alpha}(r)=\exp \left(\frac{-c}{(1-r)^{\alpha}}\right), \quad \alpha>0, c>0,
$$

and the double exponential weights

$$
w(r)=\exp \left(-\gamma \exp \left(\frac{\beta}{(1-r)^{\alpha}}\right)\right), \quad \alpha, \beta, \gamma>0 .
$$

For simplicity, we say that the Hankel operator $H_{\bar{g}}$ is bounded (or compact) on $A^{2}(w)$ when $H_{\bar{g}}: A^{2}(w) \rightarrow L^{2}(w)$ is bounded (or compact). The description of the boundedness of the Hankel operator $H_{\bar{g}}$ on $A^{2}(w)$ is stated below.

Theorem 1. Let $w \in \mathcal{W}$ and $g \in A^{2}(w)$. Then $H_{\bar{g}}$ is bounded on $A^{2}(w)$ if and only if

$$
\sup _{z \in \mathbf{D}} \tau(z)\left|g^{\prime}(z)\right|<\infty .
$$

Note that for the standards weights $\left(1-r^{2}\right)^{\alpha}$ with $\alpha>-1$, the associated function $\tau(z)$ is comparable to $\left(1-|z|^{2}\right)$. Thus Theorem 1 generalizes the well known results of Axler [5] and Arazy-Fisher-Peetre [3] on the boundedness of $H_{\bar{g}}$ on standard Bergman spaces. 
If $g \in H(\mathbf{D})$, the integration operator $J_{g}$ is defined as

$$
J_{g} f(z)=\int_{0}^{z} f(\zeta) g^{\prime}(\zeta) d \zeta, \quad f \in H(\mathbf{D}) .
$$

The boundedness, compactness and membership in Schatten $p$-classes of Hankel operators with conjugate analytic symbols and integration operators on Hardy and standard Bergman spaces are described by the same conditions (see [3], [1], [2], [5], [12], [17] and [20]). So, a natural question of Dyakonov [11] is to ask if this is always the case, that is, if the operators $J_{g}$ and $H_{\bar{g}}$ always have the same behavior. Here we will answer Dyakonov's question in the negative. Consider the exponential-type weights

$$
w_{\alpha}(z)=\exp \left(-\frac{1}{(1-|z|)^{\alpha}}\right), \quad \alpha>0 .
$$

From [9] and [19], we know that $J_{g}$ is bounded on $A^{2}\left(w_{\alpha}\right)$ if and only if

$$
\sup _{z \in \mathbf{D}}\left(1-|z|^{2}\right)^{1+\alpha}\left|g^{\prime}(z)\right|<\infty .
$$

On the other hand, since for the weight $w_{\alpha}$ one has $\tau(z) \asymp\left(1-|z|^{2}\right)^{1+\frac{\alpha}{2}}$ (see [19, Example 1]), it follows from Theorem 1 that the Hankel operator $H_{\bar{g}}$ is bounded on $A^{2}\left(w_{\alpha}\right)$ if and only if

$$
\sup _{z \in \mathbf{D}}\left(1-|z|^{2}\right)^{1+\frac{\alpha}{2}}\left|g^{\prime}(z)\right|<\infty .
$$

Therefore, for the analytic function

$$
g(z)=\frac{1}{(1-z)^{\alpha}}, \quad z \in \mathbf{D}
$$

the integration operator $J_{g}$ is bounded on $A^{2}\left(w_{\alpha}\right)$, but the Hankel operator $H_{\bar{g}}$ is unbounded on $A^{2}\left(w_{\alpha}\right)$.

The corresponding result for compactness of the Hankel operator $H_{\bar{g}}$ on $A^{2}(w)$ is the following one.

Theorem 2. Let $w \in \mathcal{W}$ and $g \in A^{2}(w)$. Then $H_{\bar{g}}$ is compact on $A^{2}(w)$ if and only if

$$
\lim _{|z| \rightarrow 1^{-}} \tau(z)\left|g^{\prime}(z)\right|=0
$$

Working a little bit more, one can get an estimate for the essential norm of the Hankel operator $H_{\bar{g}}$ on $A^{2}(w)$. The essential norm of a bounded linear operator $T$ is defined to be the distance to the compact operators, that is,

$$
\|T\|_{e}=\inf \{\|T-K\|: K \text { is compact }\} .
$$

Theorem 3. Let $w \in \mathcal{W}$ and $g \in A^{2}(w)$ with $H_{\bar{g}}$ bounded on $A^{2}(w)$. Then

$$
\left\|H_{\bar{g}}\right\|_{e} \asymp \limsup _{|a| \rightarrow 1^{-}} \tau(a)\left|g^{\prime}(a)\right| \text {. }
$$

Of course, since $\|T\|_{e}=0$ if and only if $T$ is compact, Theorem 2 is just a corollary of Theorem 3, but for convenience of the reader we find more instructive to prove Theorem 2 first without using the concept of essential norm.

Also, if $H$ and $\mathcal{K}$ are separable Hilbert spaces, a compact operator $T$ from $H$ to $\mathcal{K}$ is said to belong to the class $\mathcal{S}_{p}$ if its sequence of singular numbers is in the sequence space $\ell^{p}$. Recall that the singular numbers of a compact operator $T$ are the 
square root of the eigenvalues of the positive operator $T^{*} T$, where $T^{*}$ denotes the adjoint of $T$. Also, the compact operator $T$ admits a decomposition of the form

$$
T=\sum_{n} \lambda_{n}\left\langle\cdot, e_{n}\right\rangle_{H} f_{n}
$$

where $\left\{\lambda_{n}\right\}$ are the singular numbers of $T,\left\{e_{n}\right\}$ is an orthonormal set in $H$, and $\left\{f_{n}\right\}$ is an orthonormal set in $\mathcal{K}$. Moreover, if the singular values $\left\{\lambda_{n}\right\}$ are ordered in a decreasing order, then

$$
\lambda_{n}=\lambda_{n}(T)=\inf \{\|T-K\|: \operatorname{rank} K \leq n\} .
$$

For $p \geq 1$, the class $\mathcal{S}_{p}$ is a Banach space with the norm $\|T\|_{p}=\left(\sum_{n}\left|\lambda_{n}\right|^{p}\right)^{1 / p}$, while for $0<p<1$ one has the inequality $\|S+T\|_{p}^{p} \leq\|S\|_{p}^{p}+\|T\|_{p}^{p}$. We refer to [29, Chapter 1] for a brief account on the theory of Schatten $p$-classes. The next result completely describes the membership in $\mathcal{S}_{p}$ of the Hankel operator $H_{\bar{g}}$ on $A^{2}(w)$.

Theorem 4. Let $w \in \mathcal{W}$ and $g \in A^{2}(w)$. Then

(i) If $1<p<\infty$, then $H_{\bar{g}}: A^{2}(w) \rightarrow L^{2}(w)$ belongs to $\mathcal{S}_{p}$ if and only if

$$
\tau g^{\prime} \in L^{p}(\mathbf{D}, \Delta \varphi d m) .
$$

(ii) If $0<p \leq 1$, then $H_{\bar{g}}: A^{2}(w) \rightarrow L^{2}(w)$ belongs to $\mathcal{S}_{p}$ if and only if $g$ is constant.

It is worth mentioning that analogous results for weighted Fock spaces had been obtained recently by Constantin and Ortega-Cerdà in [7], and by Seip and Youssfi in [23]. However, in the Fock space setting, the corresponding Hankel operator is never Hilbert-Schmidt (unless the symbol is constant), so that they only need to deal with the easiest case $p>2$ in their description of Schatten classes Hankel operators on weighted Fock spaces.

Throughout the paper, the letter $C$ will denote an absolute constant whose value may change at different occurrences. We write $A \asymp B$ when the two quantities $A$ and $B$ are comparable. The paper is organized as follows: Section 2 is devoted to some required preliminaries. The proofs of Theorems 1, 2 and 3 are given in Section 3, and in Section 4 we prove Theorem 4.

\section{Preliminaries}

In this section we provide the basic tools for the proofs of the main results of the paper. For $a \in \mathbf{D}$ and $\delta>0$, we use the notation $D(\delta \tau(a))$ for the euclidian disc centered at $a$ and radius $\delta \tau(a)$. It is straightforward to see that if a weight $w$ belongs to the class $\mathcal{W}$, then its associated function $\tau(z)$ satisfies the following two properties:

(a) There is a constant $C_{1}$ such that $\tau(z) \leq C_{1}(1-|z|)$ for all $z \in \mathbf{D}$;

(b) There is a constant $C_{2}$ such that $|\tau(z)-\tau(\zeta)| \leq C_{2}|z-\zeta|$ for all $z, \zeta \in \mathbf{D}$.

A positive function $\tau$ on $\mathbf{D}$ satisfying the above two properties is said to belong to the class $\mathcal{L}$. It is very easy to see $([19$, Lemma 2.1]) that if $\tau \in \mathcal{L}$, then

$$
\tau(z) \asymp \tau(a) \text { if } z \in D(\delta \tau(a))
$$

for sufficiently small $\delta>0$. This fact will be used repeatedly throughout the paper. 
We begin by recalling two results from [19]. The first one is some type of generalized sub-mean value property for $|f|^{p} w$ that gives the boundedness of the point evaluation functionals on $A^{2}(w)$.

Lemma A. [19, Lemma 2.2] Let $w \in \mathcal{W}$ and $a \in \mathbf{D}$. Then there exists a constant $M \geq 1$ such that

$$
|f(a)|^{2} w(a) \leq \frac{M}{\delta^{2} \tau(a)^{2}} \int_{D(\delta \tau(a))}|f(z)|^{2} w(z) d m(z),
$$

for all $f \in H(\mathbf{D})$ and all sufficiently small $\delta>0$.

Since the norm of the point evaluation functional equals the norm of the reproducing kernel in $A^{2}(w)$, this result also gives an upper bound for $\left\|K_{z}\right\|_{w}$. The next result says that this upper bound is the corresponding growth of the reproducing kernel.

Lemma B. [19] If $w$ is a weight in the class $\mathcal{W}$, then

$$
\left\|K_{z}\right\|_{w}^{2} w(z) \asymp \tau(z)^{-2} \asymp \Delta \varphi(z) .
$$

The next result we need is an estimate of the reproducing kernel function for points close to the diagonal. Despite that this result is stated in [15, Lemma 3.6] for a class of weights that does not include the double exponential weights, the same proof works for weights in the class $\mathcal{W}$.

Lemma C. Let $w \in \mathcal{W}$ and $z \in \mathbf{D}$. For $\delta>0$ sufficiently small we have

$$
\left|K_{z}(u)\right| \asymp\left\|K_{z}\right\|_{w}\left\|K_{u}\right\|_{w}
$$

if $u \in D(\delta \tau(z))$.

We will also use the following lemma on coverings due to Oleinik, see [18].

Lemma D. Let $\tau$ be a positive function in $\mathbf{D}$ in the class $\mathcal{L}$, and let $\delta>0$ be sufficiently small. Then there exists a sequence of points $\left\{z_{j}\right\} \subset \mathbf{D}$, such that the following conditions are satisfied:

(i) $z_{j} \notin D\left(\delta \tau\left(z_{k}\right)\right), j \neq k$.

(ii) $\bigcup_{j} D\left(\delta \tau\left(z_{j}\right)\right)=\mathbf{D}$.

(iii) $\widetilde{D}\left(\delta \tau\left(z_{j}\right)\right) \subset D\left(3 \delta \tau\left(z_{j}\right)\right)$, where

$$
\widetilde{D}\left(\delta \tau\left(z_{j}\right)\right)=\bigcup_{z \in D\left(\delta \tau\left(z_{j}\right)\right)} D(\delta \tau(z)), \quad j=1,2, \ldots
$$

(iv) $\left\{D\left(3 \delta \tau\left(z_{j}\right)\right)\right\}$ is a covering of $\mathbf{D}$ of finite multiplicity $N$.

We shall also need the following elementary result.

Lemma 2.1. Let $1 \leq p<\infty, g \in H(\mathbf{D})$ and $a \in \mathbf{D}$. Then

$$
\tau(a)\left|g^{\prime}(a)\right| \leq C\left(\frac{1}{\tau(a)^{2}} \int_{D(\delta \tau(a))}|g(z)-g(a)|^{p} d m(z)\right)^{1 / p} .
$$

Proof. By Cauchy's formula,

$$
g^{\prime}(a)=\frac{1}{2 \pi} \int_{0}^{2 \pi} \frac{g\left(a+r e^{i \theta}\right)-g(a)}{r e^{i \theta}} d \theta
$$


and integrating both sides in the variable $r$ from $\frac{\delta}{2} \tau(a)$ to $\delta \tau(a)$ yields

$$
\tau(a) g^{\prime}(a)=\frac{4}{3 \delta^{2} \tau(a)} \int_{A(\delta \tau(a))} \frac{g(z)-g(a)}{z-a} d m(z),
$$

where $A(\delta \tau(a))$ is the annulus $D(a, \delta \tau(a)) \backslash D\left(a, \frac{\delta}{2} \tau(a)\right)$. Now, since $|z-a| \geq \frac{\delta}{2} \tau(a)$ for $z \in A(\delta \tau(a))$, if $p=1$ we are done, and the result for $p>1$ follows from Hölder's inequality.

We will also use the fact that for any orthonormal set $\left\{e_{n}\right\}$ of a reproducing kernel Hilbert space $H$, one has

$$
\sum_{n}\left|e_{n}(z)\right|^{2} \leq\left\|K_{z}\right\|_{H}^{2}, \quad z \in \mathbf{D}
$$

with equality if $\left\{e_{n}\right\}$ is also an orthonormal basis. Here $K_{z}$ is the reproducing kernel function of $H$.

\section{Bounded and compact Hankel operators}

3.1. Proof of Theorem 1. Suppose first that $H_{\bar{g}}$ is bounded on $A^{2}(w)$. Then

$$
\sup _{a \in \mathbf{D}}\left\|H_{\bar{g}} k_{a}\right\|_{w} \leq\left\|H_{\bar{g}}\right\|
$$

where $k_{a}$ are the normalized reproducing kernels of $A^{2}(w)$. Let $g_{z}(\zeta)=(g(z)-$ $g(\zeta)) K_{z}(\zeta)$. Then

$$
\begin{aligned}
H_{\bar{g}} k_{a}(z) & =\int_{\mathbf{D}} \overline{g(z)-g(\zeta)} k_{a}(\zeta) \overline{K_{z}(\zeta)} w(\zeta) d m(\zeta) \\
& =\left\|K_{a}\right\|_{w}^{-1} \overline{\left\langle g_{z}, K_{a}\right\rangle_{w}}=\left\|K_{a}\right\|_{w}^{-1} \overline{g_{z}(a)},
\end{aligned}
$$

A remark must be made here on the identity $g_{z}(a)=\left\langle g_{z}, K_{a}\right\rangle_{w}$ that holds initially only if $g_{z} \in A^{2}(w)$, and this will be the case if one is able to show that $\left|K_{z}(\zeta)\right| \leq C_{z}$ for all $\zeta \in \mathbf{D}$ for some constant $C_{z}$ depending only on $z$. But, in any case, the reproducing formula

$$
f(a)=\left\langle f, K_{a}\right\rangle_{w}=\int_{\mathbf{D}} f(\zeta) \overline{K_{a}(\zeta)} w(\zeta) d m(\zeta), \quad a \in \mathbf{D}
$$

extends to all functions $f$ being in $A^{1}(w):=L^{1}(\mathbf{D}, w d m) \cap H(\mathbf{D})$ due to the density of $A^{2}(w)$ in $A^{1}(w)$, and by Cauchy-Schwarz inequality the function $g_{z}$ clearly belongs to $A^{1}(w)$. Therefore, (3.1) together with the fact that $\left|K_{z}(a)\right| \asymp\left\|K_{z}\right\|_{w}\left\|K_{a}\right\|_{w}$ for $z \in D(\delta \tau(a))$ if $\delta>0$ is sufficiently small (see Lemma C), gives

$$
\begin{aligned}
\left\|H_{\bar{g}} k_{a}\right\|_{w}^{2} & =\left\|K_{a}\right\|_{w}^{-2} \int_{\mathbf{D}}\left|g_{z}(a)\right|^{2} w(z) d m(z) \\
& =\left\|K_{a}\right\|_{w}^{-2} \int_{\mathbf{D}}|g(z)-g(a)|^{2}\left|K_{z}(a)\right|^{2} w(z) d m(z) \\
& \geq\left\|K_{a}\right\|_{w}^{-2} \int_{D(\delta \tau(a))}|g(z)-g(a)|^{2}\left|K_{z}(a)\right|^{2} w(z) d m(z) \\
& \asymp \int_{D(\delta \tau(a))}|g(z)-g(a)|^{2}\left\|K_{z}\right\|_{w}^{2} w(z) d m(z) .
\end{aligned}
$$


Now, using that $\left\|K_{z}\right\|_{w}^{2} w(z) \asymp \tau(z)^{-2}$ (see Lemma B), and the fact that $\tau(z) \asymp \tau(a)$ for $z \in D(\delta \tau(a))$, we obtain

$$
\sup _{a \in \mathbf{D}} \frac{1}{\tau(a)^{2}} \int_{D(\delta \tau(a))}|g(z)-g(a)|^{2} d m(z)<\infty .
$$

Finally, by Lemma 2.1, this gives

$$
\sup _{a \in \mathbf{D}} \tau(a)\left|g^{\prime}(a)\right|<\infty .
$$

For the converse we need the following classical result of Hörmander (see [14, Lemma 4.4.1]) on $L^{2}$ estimates of solutions of the $\bar{\partial}$-equation.

Lemma E. [Hörmander] Let $\varphi \in C^{2}(\mathbf{D})$ with $\Delta \varphi>0$ on $\mathbf{D}$. Then there is $u \in L^{2}\left(\mathbf{D}, e^{-\varphi} d m\right)$ with $\bar{\partial} u=f$ such that

$$
\int_{\mathbf{D}}|u(z)|^{2} e^{-\varphi(z)} d m(z) \leq C \int_{\mathbf{D}}|f(z)|^{2} \frac{e^{-\varphi(z)}}{\Delta \varphi(z)} d m(z)
$$

provided the right hand side integral is finite.

Suppose that $\sup _{a \in \mathbf{D}} \tau(a)\left|g^{\prime}(a)\right|<\infty$. Note that, for $f \in H^{\infty}$, one has that $H_{\bar{g}} f=\bar{g} f-P(\bar{g} f)$ is the solution of the $\bar{\partial}$-equation $\bar{\partial} h=\overline{g^{\prime}} f$ with minimal $L^{2}(w)$ norm. By Hörmander's theorem, there is a solution $h$ with

$$
\begin{aligned}
\|h\|_{w}^{2} & \leq C \int_{\mathbf{D}}\left|g^{\prime}(z)\right|^{2}|f(z)|^{2} \frac{w(z)}{\Delta \varphi(z)} d m(z) \\
& \asymp \int_{\mathbf{D}}\left(\tau(z)\left|g^{\prime}(z)\right|\right)^{2}|f(z)|^{2} w(z) d m(z) \\
& \leq C\|f\|_{w}^{2} .
\end{aligned}
$$

Thus $\left\|H_{\bar{g}} f\right\|_{w} \leq\|h\|_{w} \leq C\|f\|_{w}$ proving that $H_{\bar{g}}$ is bounded on $A^{2}(w)$.

3.2. Proof of Theorem 2. Suppose first that $H_{\bar{g}}$ is compact on $A^{2}(w)$. Since $\left\{k_{a}\right\}_{a \in \mathbf{D}}$ is a bounded set on $A^{2}(w)$, it follows that $\left\{H_{\bar{g}} k_{a}\right\}_{a \in \mathbf{D}}$ is relatively compact on $L^{2}(w)$. Thus, by the Kolmogorov-Riesz-Tamarkin compactness theorem,

$$
\lim _{r \rightarrow 1^{-}} \int_{r<|z|<1}\left|H_{\bar{g}} k_{a}(z)\right|^{2} w(z) d m(z)=0
$$

uniformly in $a$. Let $\delta>0$ be sufficiently small. By Lemma 2.1 and (3.1), we get

$$
\begin{aligned}
\tau(a)\left|g^{\prime}(a)\right| & \leq C\left(\frac{1}{\tau(a)^{2}} \int_{D(\delta \tau(a))}|g(z)-g(a)|^{2} d m(z)\right)^{1 / 2} \\
& \leq C\left(\int_{D(\delta \tau(a))}\left|H_{\bar{g}} k_{a}(z)\right|^{2} w(z) d m(z)\right)^{1 / 2}
\end{aligned}
$$

which, by (3.3), tends to zero as $|a| \rightarrow 1^{-}$.

Conversely, suppose that $\tau(z)\left|g^{\prime}(z)\right| \rightarrow 0$ as $|z| \rightarrow 1^{-}$. Let $\left(f_{n}\right)$ be a bounded sequence in $A^{2}(w)$ converging to zero uniformly on compact subsets of $\mathbf{D}$. By the assumption, given any $\varepsilon>0$, there is $0<r_{0}<1$ such that

$$
\tau(z)\left|g^{\prime}(z)\right|<\varepsilon, \quad|z|>r_{0} .
$$


Since $\left(f_{n}\right)$ converges to zero uniformly on compact subsets of $\mathbf{D}$, we can choose a positive integer $n_{0}$ such that $\left|f_{n}(z)\right|<\varepsilon$, for $|z| \leq r_{0}$, and $n \geq n_{0}$. Thus, by (3.2) in the proof of Theorem 1,

$$
\begin{aligned}
\left\|H_{\bar{g}} f_{n}\right\|_{w}^{2} & \leq C \int_{\mathbf{D}}\left(\tau(z)\left|g^{\prime}(z)\right|\right)^{2}\left|f_{n}(z)\right|^{2} w(z) d m(z) \\
& \leq C\left(\int_{|z| \leq r_{0}}+\int_{|z|>r_{0}}\right)\left(\tau(z)\left|g^{\prime}(z)\right|\right)^{2}\left|f_{n}(z)\right|^{2} w(z) d m(z) \\
& \leq C \varepsilon^{2}\left(\sup _{z \in \mathbf{D}} \tau(z)\left|g^{\prime}(z)\right|\right)^{2}+C \varepsilon^{2}\left\|f_{n}\right\|_{w}^{2}
\end{aligned}
$$

for all $n \geq n_{0}$. This shows that $\left\|H_{\bar{g}} f_{n}\right\|_{w} \rightarrow 0$ as $n \rightarrow \infty$, and it is standard that this implies that $H_{\bar{g}}$ is compact on $A^{2}(w)$ (see, for example, the proof of Proposition 3.11 in $[8])$.

3.3. Proof of Theorem 3. Note first that the normalized reproducing kernels $k_{a}$ converges weakly to zero as $|a| \rightarrow 1^{-}$. Indeed, since $\left|\left\langle f, k_{a}\right\rangle_{w}\right|=\left\|K_{a}\right\|_{w}^{-1}|f(a)|$ for any $f \in A^{2}(w)$, this is a straightforward consequence of Lemma $\mathrm{A}$, Lemma B and the density of the polynomials on $A^{2}(w)$. Thus, if $K$ is any compact operator from $A^{2}(w)$ to $L^{2}(w)$, then $\lim _{|a| \rightarrow 1^{-}}\left\|K\left(k_{a}\right)\right\|_{w}=0$, and this gives

$$
\left\|H_{\bar{g}}-K\right\| \geq \limsup _{|a| \rightarrow 1^{-}}\left\|H_{\bar{g}} k_{a}-K\left(k_{a}\right)\right\|_{w} \geq \limsup _{|a| \rightarrow 1^{-}}\left\|H_{\bar{g}} k_{a}\right\|_{w} .
$$

Therefore, if $\delta>0$ is sufficiently small, arguing as in the proof of Theorem 2 one obtains

$$
\begin{aligned}
\left\|H_{\bar{g}}\right\|_{e} & \geq \limsup _{|a| \rightarrow 1^{-}}\left\|H_{\bar{g}} k_{a}\right\|_{w} \\
& \geq \limsup _{|a| \rightarrow 1^{-}}\left(\int_{D(\delta \tau(a))}\left|H_{\bar{g}} k_{a}(z)\right|^{2} w(z) d m(z)\right)^{1 / 2} \\
& \geq C \limsup _{|a| \rightarrow 1^{-}} \tau(a)\left|g^{\prime}(a)\right| .
\end{aligned}
$$

This proves the lower estimate. In order to obtain the upper estimate, note first that the operator $K_{r}$ defined by $K_{r}(f)(z)=f(r z)$ is a compact operator on $A^{2}(w)$ for any $0<r<1$ (it is straightforward to see that if $\left\{f_{n}\right\}$ is a bounded sequence in $A^{2}(w)$ converging to zero uniformly on compact subsets of $\mathbf{D}$, then $\lim _{n}\left\|K_{r} f_{n}\right\|_{w}=0$ ). Thus, $H_{\bar{g}} K_{r}$ is a compact operator from $A^{2}(w)$ to $L^{2}(w)$, and therefore

$$
\left\|H_{\bar{g}}\right\|_{e} \leq\left\|H_{\bar{g}}-H_{\bar{g}} K_{r}\right\| \text {. }
$$

Now take $\left\{r_{n}\right\} \subset(0,1)$ with $r_{n} \rightarrow 1$. For $f \in H^{\infty}$ with $\|f\|_{w} \leq 1$, by (3.2) in the proof of Theorem 1, we have

$$
\begin{aligned}
\left\|\left(H_{\bar{g}}-H_{\bar{g}} K_{r_{n}}\right)(f)\right\|_{w} & =\left\|H_{\bar{g}}\left(I-K_{r_{n}}\right)(f)\right\|_{w} \\
& \leq C\left(\int_{\mathbf{D}} \tau(z)^{2}\left|g^{\prime}(z)\right|^{2}\left|\left(I-K_{r_{n}}\right)(f)(z)\right|^{2} w(z) d m(z)\right)^{1 / 2} \\
& =C\left(\left(\int_{|z| \leq t}+\int_{|z|>t}\right) \tau(z)^{2}\left|g^{\prime}(z)\right|^{2}\left|f_{n}(z)\right|^{2} w(z) d m(z)\right)^{1 / 2},
\end{aligned}
$$

where $0<t<1$ is fixed, and $f_{n}(z)=\left(I-K_{r_{n}}\right)(f)(z)=f(z)-f\left(r_{n} z\right)$. Since $\left\{f_{n}\right\}$ converges to zero uniformly on compact subsets of $\mathbf{D}$, and $\sup _{z \in \mathbf{D}} \tau(z)\left|g^{\prime}(z)\right|<\infty$ 
due to the boundedness of $H_{\bar{g}}$ (see Theorem 1), one has

$$
\int_{|z| \leq t} \tau(z)^{2}\left|g^{\prime}(z)\right|^{2}\left|f_{n}(z)\right|^{2} w(z) d m(z) \rightarrow 0 \quad \text { as } \quad n \rightarrow \infty .
$$

This, together with the fact that $\left\|f_{n}\right\|_{w} \leq C$, gives

$$
\left\|H_{\bar{g}}\right\|_{e} \leq C \sup _{|z|>t} \tau(z)\left|g^{\prime}(z)\right|
$$

and letting $t$ to tend to 1 , we obtain the upper estimate completing the proof.

\section{Schatten class Hankel operators: Proof of Theorem 4}

4.1. Proof of Sufficiency. Due to (3.2), one has the inequality

$$
\left\|H_{\bar{g}} f\right\|_{w} \leq C\left\|M_{\tau g^{\prime}} f\right\|_{w},
$$

and thus it is enough to show that the multiplication operator $M_{\tau g^{\prime}}: A^{2}(w) \rightarrow L^{2}(w)$ belongs to $\mathcal{S}_{p}$. We first consider the easiest case $p \geq 2$. Let $\left\{e_{n}\right\}$ be any orthonormal set of $A^{2}(w)$. Then, by Hölder's inequality, (2.1) and Lemma B, one has

$$
\begin{aligned}
\sum_{n}\left\|M_{\tau g^{\prime}} e_{n}\right\|_{w}^{p} & =\sum_{n}\left(\int_{\mathbf{D}} \tau(z)^{2}\left|g^{\prime}(z)\right|^{2}\left|e_{n}(z)\right|^{2} w(z) d m(z)\right)^{p / 2} \\
& \leq \sum_{n} \int_{\mathbf{D}} \tau(z)^{p}\left|g^{\prime}(z)\right|^{p}\left|e_{n}(z)\right|^{2} w(z) d m(z) \\
& =\int_{\mathbf{D}} \tau(z)^{p}\left|g^{\prime}(z)\right|^{p}\left(\sum_{n}\left|e_{n}(z)\right|^{2}\right) w(z) d m(z) \\
& \leq \int_{\mathbf{D}} \tau(z)^{p}\left|g^{\prime}(z)\right|^{p}\left\|K_{z}\right\|_{w}^{2} w(z) d m(z) \\
& \leq C \int_{\mathbf{D}} \tau(z)^{p}\left|g^{\prime}(z)\right|^{p} \Delta \varphi(z) d m(z) .
\end{aligned}
$$

Thus, by [29, Theorem 1.27], the operator $M_{\tau g^{\prime}}$ belongs to $\mathcal{S}_{p}$.

The case $1<p<2$ is a little bit more involved. The first step is to show that $M_{\tau g^{\prime}}: A^{2}(w) \rightarrow L^{2}(w)$ belongs to $\mathcal{S}_{p}$ if and only if the multiplication operator $M_{g^{\prime}}: A^{2}(w) \rightarrow A^{2}\left(w^{*}\right)$ is in $\mathcal{S}_{p}$, where $w^{*}$ is the weight function

$$
w^{*}(z)=\tau(z)^{2} w(z) .
$$

To see that, since $M_{\tau g^{\prime}}$ is in $\mathcal{S}_{p}$ if and only if $S:=M_{\tau g^{\prime}}^{*} M_{\tau g^{\prime}}: A^{2}(w) \rightarrow A^{2}(w)$ is in $\mathcal{S}_{p / 2}$, and $M_{g^{\prime}} \in \mathcal{S}_{p}$ if and only if $T:=M_{g^{\prime}}^{*} M_{g^{\prime}}: A^{2}(w) \rightarrow A^{2}(w)$ belongs to $\mathcal{S}_{p / 2}$, it is enough to see that the operators $S$ and $T$ are the same. Let $\left\{e_{n}\right\}$ be any orthonormal basis of $A^{2}(w)$ and let $h \in A^{2}(w)$. Then

$$
\begin{aligned}
\left\langle S e_{n}, h\right\rangle_{w} & =\left\langle M_{\tau g^{\prime}} e_{n}, M_{\tau g^{\prime}} h\right\rangle_{w}=\left\langle\tau g^{\prime} e_{n}, \tau g^{\prime} h\right\rangle_{w} \\
& =\int_{\mathbf{D}}\left|g^{\prime}(z)\right|^{2} e_{n}(z) \overline{h(z)} \tau(z)^{2} w(z) d m(z) \\
& =\int_{\mathbf{D}}\left|g^{\prime}(z)\right|^{2} e_{n}(z) \overline{h(z)} w^{*}(z) d m(z) \\
& =\left\langle M_{g^{\prime}} e_{n}, M_{g^{\prime}} h\right\rangle_{w^{*}}=\left\langle T e_{n}, h\right\rangle_{w} .
\end{aligned}
$$


This shows that $S e_{n}=T e_{n}$ for any $n$ and therefore the operators $S$ and $T$ are the same. So, assume that $\tau g^{\prime} \in L^{p}(\mathbf{D}, \Delta \varphi d m)$ and let's proceed to show that the multiplication operator $M_{g^{\prime}}: A^{2}(w) \rightarrow A^{2}\left(w^{*}\right)$ belongs to $\mathcal{S}_{p}$. Let $\left\{e_{n}\right\}$ and $\left\{\sigma_{n}\right\}$ be orthonormal sets in $A^{2}(w)$ and $A^{2}\left(w^{*}\right)$ respectively. Since $\left\|\sigma_{n}\right\|_{w^{*}}=1$, an application of Hölder's inequality gives

$$
\begin{aligned}
\sum_{n}\left|\left\langle M_{g^{\prime}} e_{n}, \sigma_{n}\right\rangle_{w^{*}}\right|^{p} & \leq \sum_{n}\left(\int_{\mathbf{D}}\left|g^{\prime}(z)\right|\left|e_{n}(z)\right|\left|\sigma_{n}(z)\right| w^{*}(z) d A(z)\right)^{p} \\
& \leq \sum_{n} \int_{\mathbf{D}}\left|g^{\prime}(z)\right|^{p}\left|e_{n}(z)\right|^{p}\left|\sigma_{n}(z)\right|^{2-p} w^{*}(z) d m(z) \\
& =\int_{\mathbf{D}}\left|g^{\prime}(z)\right|^{p}\left(\sum_{n}\left|e_{n}(z)\right|^{p}\left|\sigma_{n}(z)\right|^{2-p}\right) w^{*}(z) d m(z) .
\end{aligned}
$$

Now, since $p<2$, Hölder's inequality with exponent $2 / p>1$ and (2.1) yields

$$
\sum_{n}\left|e_{n}(z)\right|^{p}\left|\sigma_{n}(z)\right|^{2-p} \leq\left(\sum_{n}\left|e_{n}(z)\right|^{2}\right)^{\frac{p}{2}}\left(\sum_{n}\left|\sigma_{n}(z)\right|^{2}\right)^{\frac{2-p}{2}} \leq\left\|K_{z}\right\|_{w}^{p}\left\|K_{z}^{*}\right\|_{w^{*}}^{2-p}
$$

where $K_{z}^{*}$ is the reproducing kernel of $A^{2}\left(w^{*}\right)$. Since, by Lemma B, we have

$$
\left\|K_{z}\right\|_{w}^{2} w(z) \asymp \tau(z)^{-2}
$$

if we are able to obtain the estimate

$$
\left\|K_{z}^{*}\right\|_{w^{*}}^{2} w^{*}(z) \leq C \tau(z)^{-2}
$$

for some positive constant $C$, we will obtain

$$
\begin{aligned}
\sum_{n}\left|\left\langle M_{g^{\prime}} e_{n}, \sigma_{n}\right\rangle_{w^{*}}\right|^{p} & \leq \int_{\mathbf{D}}\left|g^{\prime}(z)\right|^{p}\left\|K_{z}\right\|_{w}^{p}\left\|K_{z}^{*}\right\|_{w^{*}}^{2-p} w^{*}(z) d m(z) \\
& \leq C \int_{\mathbf{D}}\left|g^{\prime}(z)\right|^{p} w(z)^{-p / 2} w^{*}(z)^{p / 2} \frac{d m(z)}{\tau(z)^{2}} \\
& =C \int_{\mathbf{D}} \tau(z)^{p}\left|g^{\prime}(z)\right|^{p} \frac{d m(z)}{\tau(z)^{2}},
\end{aligned}
$$

and this will prove that the multiplication operator $M_{g^{\prime}}: A^{2}(w) \rightarrow A^{2}\left(w^{*}\right)$ belongs to $\mathcal{S}_{p}$ finishing the proof of the sufficiency. Note that (4.1) does not follow directly from Lemma $\mathrm{B}$, since it is not clear if the weight $w^{*}$ belongs to the class $\mathcal{W}$. To see why the inequality (4.1) is true, let $f \in A^{2}\left(w^{*}\right)$ and use Lemma $\mathrm{A}$ in order to obtain

$$
|f(z)|^{2} w(z) \leq \frac{C}{\tau(z)^{2}} \int_{D(\delta \tau(z))}|f(u)|^{2} w(u) d m(u) .
$$

This together with the fact that $\tau(u) \asymp \tau(z)$ if $u \in D(\delta \tau(z))$ yields

$$
|f(z)|^{2} w^{*}(z)=\tau(z)^{2}|f(z)|^{2} w(z) \leq \frac{C}{\tau(z)^{2}} \int_{D(\delta \tau(z))}|f(u)|^{2} w^{*}(u) d m(u) .
$$

Since $\left\|K_{z}^{*}\right\|_{w^{*}}$ coincides with the norm of the point evaluation functional in $A^{2}\left(w^{*}\right)$ at the point $z$, this establishes (4.1) completing the proof.

4.2. Proof of necessity. Suppose that $H_{\bar{g}} \in \mathcal{S}_{p}$. We first deal with the case $p \geq 2$. The method of proof for this case is standard (see [24] for example). Since $H_{\bar{g}}$ 
is compact, there are orthonormal sets $\left\{e_{n}\right\}$ and $\left\{\sigma_{n}\right\}$ of $A^{2}(w)$ and $L^{2}(w)$ respectively such that

$$
H_{\bar{g}} f=\sum_{n} \lambda_{n}\left\langle f, e_{n}\right\rangle_{w} \sigma_{n}
$$

where $\left\{\lambda_{n}\right\}$ are the singular values of $H_{\bar{g}}$. Therefore

$$
H_{\bar{g}} K_{z}=\sum_{n} \lambda_{n} \overline{e_{n}(z)} \sigma_{n}
$$

and, since $p \geq 2$, it follows from Hölder's inequality and (2.1) that

$$
\begin{aligned}
\left\|H_{\bar{g}} K_{z}\right\|_{w}^{p} & =\left(\sum_{n}\left|\lambda_{n}\right|^{2}\left|e_{n}(z)\right|^{2}\right)^{p / 2} \\
& \leq\left(\sum_{n}\left|\lambda_{n}\right|^{p}\left|e_{n}(z)\right|^{2}\right)\left(\sum_{n}\left|e_{n}(z)\right|^{2}\right)^{\frac{p-2}{2}} \\
& \leq\left(\sum_{n}\left|\lambda_{n}\right|^{p}\left|e_{n}(z)\right|^{2}\right)\left\|K_{z}\right\|_{w}^{p-2} .
\end{aligned}
$$

Now, the proof of Theorem 1 gives the inequality $\tau(z)\left|g^{\prime}(z)\right| \leq C\left\|H_{\bar{g}} k_{z}\right\|_{w}$, where $k_{z}$ are the normalized reproducing kernels. This, Lemma B and (4.2) yields

$$
\begin{aligned}
\int_{\mathbf{D}} \tau(z)^{p}\left|g^{\prime}(z)\right|^{p} \Delta \varphi(z) d m(z) & \asymp \int_{\mathbf{D}} \tau(z)^{p}\left|g^{\prime}(z)\right|^{p}\left\|K_{z}\right\|_{w}^{2} w(z) d m(z) \\
& \leq C \int_{\mathbf{D}}\left\|H_{\bar{g}} k_{z}\right\|_{w}^{p}\left\|K_{z}\right\|_{w}^{2} w(z) d m(z) \\
& =C \int_{\mathbf{D}}\left\|H_{\bar{g}} K_{z}\right\|_{w}^{p}\left\|K_{z}\right\|_{w}^{2-p} w(z) d m(z) \\
& \leq C \int_{\mathbf{D}} \sum_{n}\left|\lambda_{n}\right|^{p}\left|e_{n}(z)\right|^{2} w(z) d m(z) \\
& =C\left\|H_{\bar{g}}\right\|_{S_{p}}^{p} .
\end{aligned}
$$

Finally, we prove the necessity in the case $1 \leq p<2$. Again, this case is more involved. Arguing as in the proof of Theorem 1 (using Lemma 2.1, equation (3.1), the fact that $\tau(z) \asymp \tau(\zeta)$ for $\zeta \in D(\delta \tau(z))$, Lemma B and Lemma $\mathrm{C}$ ) one can show that

$$
\begin{aligned}
\tau(z)\left|g^{\prime}(z)\right| & \leq C\left(\frac{1}{\tau(z)^{2}} \int_{D(\delta \tau(z))}|g(\zeta)-g(z)|^{p} d m(\zeta)\right)^{1 / p} \\
& \leq C\left(\int_{D(\delta \tau(z))}\left|H_{\bar{g}} k_{z}(\zeta)\right|^{p}\left\|K_{\zeta}\right\|_{w}^{2-p} w(\zeta) d m(\zeta)\right)^{1 / p}
\end{aligned}
$$

Now, since

$$
H_{\bar{g}} K_{z}(\zeta)=\sum_{n} \lambda_{n} \overline{e_{n}(z)} f_{n}(\zeta)
$$


with $\left\{\lambda_{n}\right\}$ being the singular numbers of $H_{\bar{g}}$, and $\left\{e_{n}\right\},\left\{f_{n}\right\}$ orthonormal sets of $A^{2}(w)$ and $L^{2}(w)$ respectively; an application of Hölder's inequality and (2.1) yields

$$
\begin{aligned}
\left|H_{\bar{g}} K_{z}(\zeta)\right|^{p} & \leq\left(\sum_{n}\left|\lambda_{n}\right|^{p}\left|e_{n}(z)\right|^{2-p}\left|f_{n}(\zeta)\right|^{p}\right)\left(\sum_{n}\left|e_{n}(z)\right|^{2}\right)^{p-1} \\
& \leq\left\|K_{z}\right\|_{w}^{2(p-1)} \sum_{n}\left|\lambda_{n}\right|^{p}\left|e_{n}(z)\right|^{2-p}\left|f_{n}(\zeta)\right|^{p}
\end{aligned}
$$

Note that the previous inequality trivially holds for $p=1$. Therefore, for $1 \leq p<2$, we obtain

$$
\begin{aligned}
\left\|\tau g^{\prime}\right\|_{L^{p}(\mathbf{D}, \Delta \varphi d m)}^{p} & \asymp \int_{\mathbf{D}}\left(\tau(z)\left|g^{\prime}(z)\right|\right)^{p} \frac{d m(z)}{\tau(z)^{2}} \\
& \leq C \int_{\mathbf{D}} \int_{D(\delta \tau(z))}\left|H_{\bar{g}} k_{z}(\zeta)\right|^{p}\left\|K_{\zeta}\right\|_{w}^{2-p} w(\zeta) d m(\zeta) \frac{d m(z)}{\tau(z)^{2}} \\
& \leq C \sum_{n}\left|\lambda_{n}\right|^{p} I_{n}
\end{aligned}
$$

where

$$
I_{n}:=\int_{\mathbf{D}}\left|e_{n}(z)\right|^{2-p} \int_{D(\delta \tau(z))}\left|f_{n}(\zeta)\right|^{p}\left\|K_{\zeta}\right\|_{w}^{2-p} w(\zeta) d m(\zeta)\left\|K_{z}\right\|_{w}^{p-2} \frac{d m(z)}{\tau(z)^{2}} .
$$

Since

$$
\left\|H_{\bar{g}}\right\|_{S_{p}}^{p}=\sum_{n}\left|\lambda_{n}\right|^{p}
$$

it is enough to show that $I_{n} \leq C$ for some constant $C$ not depending on $n$. Now, an application of Hölder's inequality gives

$$
I_{n} \leq\left\|e_{n}\right\|_{w}^{2-p} J_{n}^{p / 2}=J_{n}^{p / 2},
$$

where

$$
J_{n}:=\int_{\mathbf{D}}\left(\int_{D(\delta \tau(z))}\left|f_{n}(\zeta)\right|^{p}\left\|K_{\zeta}\right\|_{w}^{2-p} w(\zeta) d m(\zeta)\right)^{2 / p}\left(\left\|K_{z}\right\|_{w}^{2} w(z)\right)^{\frac{p-2}{p}} \frac{d m(z)}{\tau(z)^{4 / p}} .
$$

Since $\left\|K_{z}\right\|^{2} w(z) \asymp \tau(z)^{-2}$ (see Lemma B), we have

$$
J_{n} \asymp \int_{\mathbf{D}}\left(\int_{D(\delta \tau(z))}\left|f_{n}(\zeta)\right|^{p}\left\|K_{\zeta}\right\|_{w}^{2-p} w(\zeta) d m(\zeta)\right)^{2 / p} \frac{d m(z)}{\tau(z)^{2}} .
$$

Using Hölder's inequality once again and Lemma B, we obtain

$$
\begin{aligned}
& \int_{D(\delta \tau(z))}\left|f_{n}(\zeta)\right|^{p}\left\|K_{\zeta}\right\|_{w}^{2-p} w(\zeta) d m(\zeta) \\
& \leq\left(\int_{D(\delta \tau(z))}\left|f_{n}(\zeta)\right|^{2} w(\zeta) d m(\zeta)\right)^{p / 2}\left(\int_{D(\delta \tau(z))}\left\|K_{\zeta}\right\|_{w}^{2} w(\zeta) d m(\zeta)\right)^{\frac{2-p}{2}} \\
& \leq C\left(\int_{D(\delta \tau(z))}\left|f_{n}(\zeta)\right|^{2} w(\zeta) d m(\zeta)\right)^{p / 2}
\end{aligned}
$$

Putting this into (4.3) gives

$$
J_{n} \leq C \int_{\mathbf{D}}\left(\int_{D(\delta \tau(z))}\left|f_{n}(\zeta)\right|^{2} w(\zeta) d m(\zeta)\right) \frac{d m(z)}{\tau(z)^{2}} .
$$


Finally, if $\left\{z_{j}\right\}$ is the sequence given by Lemma D, we obtain

$$
\begin{aligned}
J_{n} & \leq C \sum_{j} \int_{D\left(\delta \tau\left(z_{j}\right)\right)}\left(\int_{D(\delta \tau(z))}\left|f_{n}(\zeta)\right|^{2} w(\zeta) d m(\zeta)\right) \frac{d m(z)}{\tau(z)^{2}} \\
& \leq C \sum_{j} \int_{\widetilde{D}\left(\delta \tau\left(z_{j}\right)\right)}\left|f_{n}(\zeta)\right|^{2} w(\zeta) d m(\zeta) \\
& \leq C \int_{\mathbf{D}}\left|f_{n}(\zeta)\right|^{2} w(\zeta) d m(\zeta)=C\left\|f_{n}\right\|_{w}^{2} \leq C
\end{aligned}
$$

This completes the proof of (i). In order to prove (ii), note that we have just proved that if $H_{\bar{g}}$ is in the trace class $\mathcal{S}_{1}$, then

$$
\int_{\mathbf{D}}\left|g^{\prime}(z)\right| \frac{d m(z)}{\tau(z)}<\infty .
$$

Since $\tau(z) \leq C(1-|z|)$, this implies that

$$
\int_{\mathbf{D}}\left|g^{\prime}(z)\right| \frac{d m(z)}{1-|z|}<\infty
$$

and thus $g$ must be a constant. Since $\mathcal{S}_{p} \subset \mathcal{S}_{1}$ for $0<p<1$, we are done.

\section{References}

[1] Aleman, A., and A. Siskakis: An integral operator on $H^{p}$. - Complex Variables 28, 1995, $149-158$

[2] Aleman, A., and A. Siskakis: Integration operators on Bergman spaces. - Indiana Univ. Math. J. 46, 1997, 337-356.

[3] Arazy, J., S. Fisher, and J. Peetre: Hankel operators on weighted Bergman spaces. Amer. J. Math. 110, 1988, 989-1054.

[4] Arazy, J., S. Fisher, S. Janson, and J. Peetre: Membership of Hankel operators on the ball in unitary ideals. - J. London Math. Soc. 43, 1991, 485-508.

[5] AxleR, S.: The Bergman space, the Bloch space, and commutators of multiplication operators. - Duke Math. J. 53, 1986, 315-332.

[6] Bonsall, F. F.: Hankel operators on the Bergman space for the disk. - J. London Math. Soc. 33, 1986, 355-364.

[7] Constantin, O., and J. Ortega-Cerdà: Some spectral properties of the canonical solution operator to $\bar{\partial}$ on weighted Fock spaces. - J. Math. Anal. Appl. 377, 2011, 353-361.

[8] Cowen, C., and B. MacCluer: Composition operators on spaces of analytic functions. CRC Press, Boca Raton, 1995.

[9] Dostanic, M: Integration operators on Bergman spaces with exponential weights. - Rev. Mat. Iberoam. 23, 2007, 421-436.

[10] Duren, P., and A. Schuster: Bergman spaces. - Amer. Math. Soc., Providence, Rhode Island, 2004.

[11] Dyakonov, K.: Personal communication.

[12] Hartman, P.: On completely continuous Hankel matrices. - Proc. Amer. Math. Soc. 9, 1958, $862-866$.

[13] Hedenmalm, H., B. Korenblum, and K. Zhu: Theory of Bergman spaces. - Springer, New York, 2000. 
[14] Hörmander, L.: An introduction to complex analysis in several variables. Third revised edition. - North Holland, Amsterdam, 1990.

[15] Lin, P., and R. Rochberg: Trace ideal criteria for Toeplitz and Hankel operators on the weighted Bergman spaces with exponential type weights. - Pacific J. Math. 173, 1996, 127-146.

[16] Janson, S.: Hankel operators between Bergman spaces. - Ark. Mat. 26, 1988, 205-219.

[17] Nehari, Z.: On bounded bilinear forms. - Ann. of Math. 65, 1957, 153-162.

[18] OleiniK, V. L.: Embedding theorems for weighted classes of harmonic and analytic functions. - J. Soviet. Math. 9, 1978, 228-243.

[19] PAU, J., and J. A. PelÁEZ: Embedding theorems and integration operators on Bergman spaces with rapidly decreasing weights. - J. Funct. Anal. 259, 2010, 2727-2756.

[20] Peller, P.: Hankel operators of class $C_{p}$ and their applications (rational approximation, Gaussian processes, the problem of majorizing operators). - Mat. Sb. 41, 1982, 443-479.

[21] Peller, V.: An excursion into the theory of Hankel operators. - In: Holomorphic spaces (Berkeley, CA, 1995), Math. Sci. Res. Inst. Publ. 33, Cambridge Univ. Press, Cambridge, 1998, 65-120.

[22] Peller, V.: Hankel operators and their applications. - Springer, 2003.

[23] Seip, K., and H. Youssfi: Hankel operators on Fock spaces and related Bergman kernel estimates. - J. Geom. Anal. (to appear).

[24] Smith, M. P.: Testing Schatten class Hankel operators and Carleson embeddings via reproducing kernels. - J. London Math. Soc. 71, 2005, 172-186.

[25] Wu, Z: Hankel and Toeplitz operators on Dirichlet spaces. - Integral Equations Operator Theory 15, 1992, 503-525.

[26] Wu, Z.: Boundedness, compactness ans Schatten p-classes of Hankel operators between weighted Dirichlet spaces. - Ark. Mat. 31, 1993, 395-417.

[27] ZHU, K.: Hankel operators on the Bergman space of bounded symmetric domains. - Trans. Amer. Math. Soc. 324, 1991, 707-730.

[28] Zhu, K.: Schatten class Hankel operators on the Bergman space of the unit ball. - Amer. J. Math. 113, 1991, 147-167.

[29] ZHU, K.: Operator theory in function spaces. Second edition. - Amer. Math. Soc., Providence, Rhode Island, 2007. 